\title{
Infographic oriented management model of cyber-physical systems during a building life cycle
}

\author{
Konstantin Losev ${ }^{1, *}$, and Vitaly Chulkov ${ }^{1}$ \\ ${ }^{1}$ University "Moscow State University of Civil Engineering", Russia, 129337, Moscow, \\ Yaroslavskoye shosse, 26
}

\begin{abstract}
The study contributes to cyber-physical system (CPS) modelling during a building life cycle. The framework through which systems engineers could design and construct these CPS requires an ontology and a structure of terms. Thus, the structure of terms for modern information system, including approaches, concepts and terms, is proposed as the basis of ontology. The task of the study was to present the model of CPS management structure in accordance with the Industry 4.0 program. In order to construct it the dynamics of the relationship of terms has been given. An infographic modeling method has been used in the study. The subject of research is one of the model levels, responsible for knowledge conversion from machine-digital to human-oriented form, which will be the basis for the operator's understanding of the knowledge accumulated by the CPS, and will help to make non-trivial management decisions. The structure of terms including approaches, concepts and terms, is proposed as the basis of ontology. The dynamics of the relationship of terms is given. Model of CPS management structure is presented in accordance with the Industry 4.0 program. The "Infographic models management" level specific to the CPS is defined. Three types of infographic slides which can be issued by CPS within the domain area of construction and design are proposed.
\end{abstract}

\section{Introduction}

The successful engineering of information technology systems during a building life cycle requires the framework through which systems engineers can design and construct it. The framework should be based on the ontology and structure of terms [1]. There are several concepts and terms have been established in recent times and some of them are partially overlapped with each other and with the concepts had been defined earlier: intelligent agent (IA) and multi-agent system (MAS), embedded and hybrid systems (EHS), cyber-physical systems (CPS), Internet of things (IoT), systems of systems (SoS), product life cycle management (PLM), digital twin (DT) and information model (IM) [2]. In oder to give a model of relationships between these concepts and terms it needs to define some of it.

\footnotetext{
* Corresponding author: aljurgaitis@gmail.com
} 
Artificial intelligence as a concept were coined earlier than the others above and could be characterized as "a system's ability to correctly interpret external data, to learn from such data, and to use those learnings to achieve specific goals and tasks through flexible adaptation" [2,3]. MAS is self-organizes system and can be defined through its interacting agents (IA), which are computational entities with some degree autonomous. IA are allowed to cooperate, compete, communicate, act flexibly, and exercise control over their behavior within the frame of their goals. They may be quite simple or rather complex [4], $[5]$.

Embedded and hybrid systems are a microprocessor or microcontroller based systems, a combination of computer hardware and software, designed for a specific function or a range of functions within a larger system. Mainly it is real-time system with limited resources and high requirements for reliability and safety of operation, either fixed in capability or programmable [6]. Traditional embedded systems had been presented as integrated circuits in 1961 and, it seems, they are coming to be replaced by microprocessor systems in the first quarter of 21 st century [7].

When community have realized "that the principal challenges in embedded systems stem from their interaction with physical processes, and not from their limited resources" (Lee, Seisha 2017), the new term was coined in 2006: cyber-physical systems (CPS), which are generally referred to as an integration of computation with physical processes. This integration could be done with or without network connection [8], [9]. Therefore, cyberphysical systems are hybrid systems with embedded software coupling heterogeneous subsystems able to sense, to act and to communicate through networks [1], [9], [11]. There is elaborate definition shown CPS " is an integration of computation with physical processes, whose behaviour is defined by both cyber and physical parts of the system. Embedded computers and networks monitor and control the physical processes affect computations and vice versa." (Fitz T., Theiler M., Smarsly K., 2019).

There are following hallmark features of CPS:

"cyber capability in physical components;

networked at multi- and extreme scales;

complex in many scales;

dynamically reorganizing/ reconfiguring;

high degrees of automation;

operation must be dependable, and in some cases certified" [12],

A newer and alternative set of CPS characteristics clarifies the features above:

"directly record physical data using sensors and affect physical processes using actuators;

evaluate and save recorded data, and actively or reactively interact both with the physical and digital world;

connected with one another and in global networks via digital communication facilities

(wireless and/or wired, local and/or global);

use globally available data and services;

have a series of dedicated, multimodal human-machine interfaces." [13].

The focus on CPS networking brings us closer to another concept: Internet of Things (Ashton K. 1999). The analogs of connected devices idea one can find even 45 years ago as "embedded internet" and "pervasive computing".

"The Internet-of-things is a system of interrelated computing devices, mechanical and digital machines, objects, animals or people that are provided with unique identifiers and the ability to transfer data over a network without requiring human-to-human or human-tocomputer interaction." (TechTarget 2016) [1].

When considering a life cycle of CPS with the notice of the interrelation complexity at different time stages we come to the next modern term "SoS", which means "a system 
which results from the coupling of a number of constituent systems at some point in their life cycles."(Brook 2016). This term makes it possible to quantize the state of the CPS in time [14].

The core of CPS information support during its life cycle is a Digital Twin concept, which is dualistic in its origin.

On the one hand, DT is an information model (IM) of the already manufactured real product, which is allowed to contain information about product defects and changes and is updated regularly in the process of a product physical use; thus, IM mirrors the life cycle of a product. [15], [16].

On the other hand, DT is a simulation derived from IM has been structured on the base of sensors mounted on a real physical product, system or entity that simulates the behavior of such product, system, entity in the real world [17]. The given definitions of DT make it a main element of CPS in a life cycle.

Product Lifecycle Management (PLM) is "an integrated, information-driven approach comprised of people, processes/practices, and technology to all aspects of a product's life, from its design through manufacture, deployment and maintenance — culminating in the product's removal from service and final disposal. By trading product information for wasted time, energy, and material across the entire organization and into the supply chain, PLM drives the next generation of lean thinking". [18]

\section{Materials and Methods}

Being a set of structured and unstructured information containers (ISO 19650-1: 2018, item 3.3.8), it is considered IM method to be the basis of CPS.

DT is an industrial subset of a general term - Information Model. IM can consist of either digital and non-virtual elements.

There are 5 concepts defined in the relevant dimensions: Product Life cycle Management (PLM) concept has defined in a Life cycle dimension; Pervasive computing in Computation; Network - in Communication; Hybrid \& Embedded Systems (HES) - in Control; Information Modelling (IM)- in Method of investigation. Concepts in turn give rise to new terms have appeared at the intersection of concepts mentioned above.

Figure 1. The model of relationships between concepts:

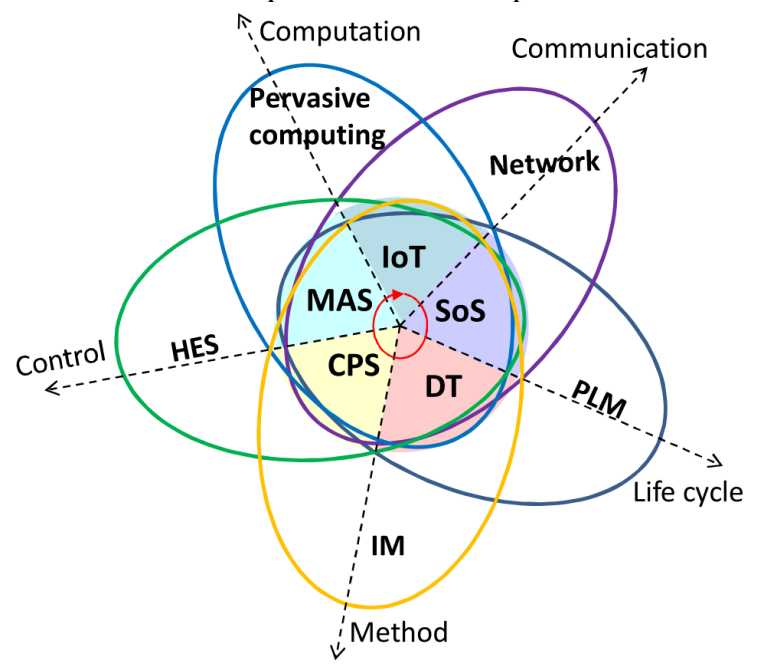

Fig. 1. An arrow on the Figure 1 shows the direction of terms flow one into other when changing a concept: 
IoT provides during CPS life cycle:

- data exchange between "Thing" and its components (entities);

- knowledge exchange (a higher type of data) between "Things" from different cyber physical systems. It's a transition to SoS.

SoS determines a dominant (focus) group of "Things" and its behavior at a particular time span. It acts partially as a temporally super CPS, and provides big data for the dominant group during its life cycle. It's a transition to DT.

DT being a virtual prototype of a real object, group of objects or processes has to be a complex software product that is created on the basis of a wide variety of data. DT of a particular "Thing" transforms big data from SoS into formal data and has been collecting the data, analyzes it and simulates behavior of real "Thing". It's a transition to CPS.

CPS correctly interprets external formal data with the help of numerous sensors on the base of physical law and physical effect software models. CPS learns from such data to achieve its goals. It's a transition to MAS.

MAS, consisting of intelligent agents (IA), transforms CPS learnings into knowledge to guarantee the achievement of specific goals and tasks through flexible adaptation and evolution. MAS brings this knowledge to the attention of each agent ("Thing") inside a particular CPS. It's a transition to IoT.

\section{Results}

The technology makes it possible to simulate a variety of situations that may arise in the workplace. Thus, the DT allows to select the most appropriate scenarios of technological processes to avoid algorithm failures and machine malfunctions. DT derived from sensor's information mounted on a physical product, so DT simulates the behavior of an product in the real world. DT is an integrated IM of the already built product, which is designed to contain and analyze information about all defects of the product and to regularly update itself throughout the life cycle. That means DT performs its functions before physical use of the product (at pre-design and design stages), during the process of the product physical use, and it retains analytical abilities even after the disposal of the product linked to the particular DT.

There is an architecture of future "Industry 4.0" CPS factories has been already suggested:

1) Smart connection (proper type and specification of sensors which provide collection of various types of data according with a specific manage and transferring protocol).

2) Data-to-information conversion (provision of self-awareness to machines by inferring meaningful information from data).

3) Cyber (machine networks forming which provide machines with current and historical self-comparison ability that allowed to predict the future behavior of the machinery).

4) Cognition (a proper info-graphics to completely transfer acquired knowledge to the experts).

5) Configuration (making machines self-configure and self-adaptive by supervisory control in a resilience control system style) [19].

It is important to compare the above architecture levels with a traditional architecture of factory automated system: 1) "Smart connection" refers to "I/O devices"; 2) "Data-toinformation conversion" - to "Programmable logic controllers (PLC)"; 3) "Cyber" - to "Supervisory control and data aquisition (SCADA)"; 4) "Cognition" - to "Manufacturing execution system (MES)"; 5) "Configuration" - to "Enterprise Resource Planning (ERP)". It is obvious that main difference between traditional and CPS architecture starts from the level 3 , because the use of SCADA does not imply full integration of computation with 
physical processes. Levels 4 and 5 are more different due to high part of artificial intelligence in CPS which leads to concentration on the proper info-graphics to a human operator and effective supervisory control from the side of the operator.

Development and expansion of enterprise subsystems "smart" behavior have forced to transfer supervisory control from the middle to the top level of system management hierarchy. It is considered to present the CPS management model in accordance with the previous suggested architecture and program "Industry 4.0" goals. The model shown on the Figure 2.

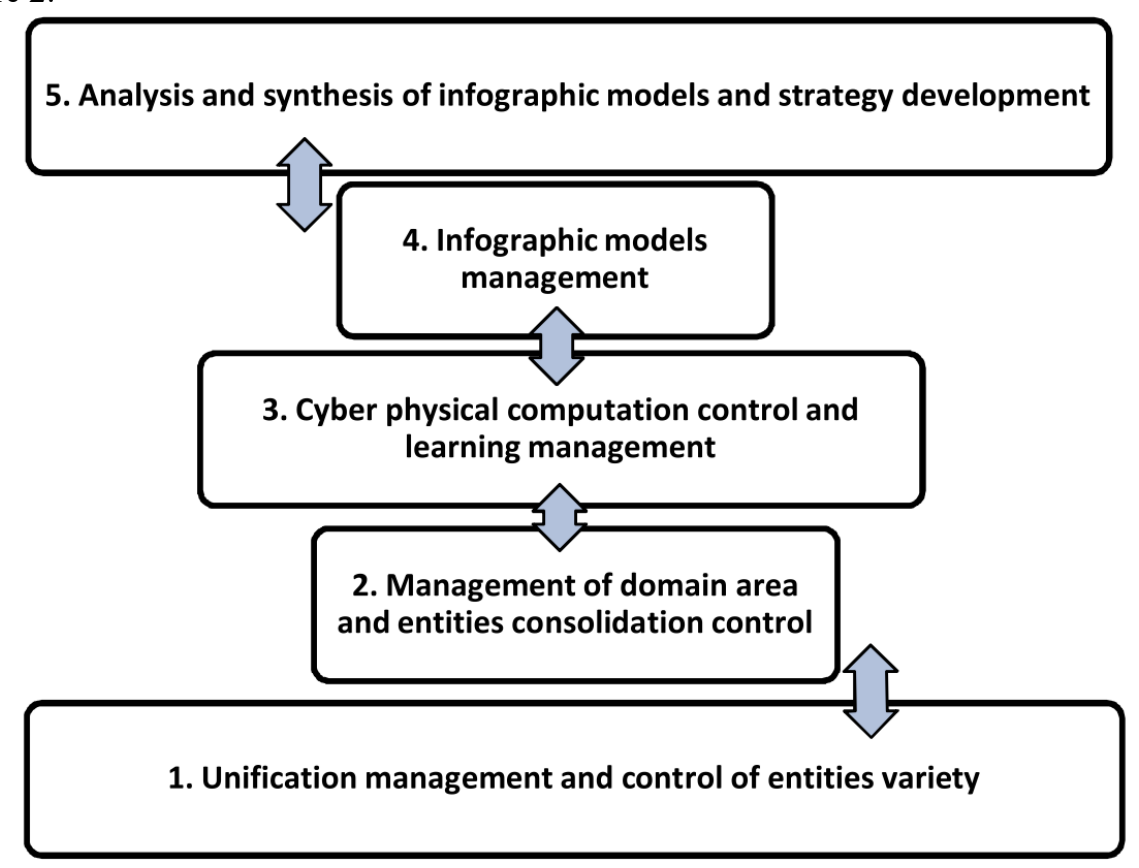

Fig. 2. Management model of a cyber-physical system

1. Unification management and control of elements variety are aimed at decreasing of hardware types and increasing of its functionality in charge of connections, data exchange protocols, reliability and compatibility (e.i. USB Type-C approach).

2. Management of domain area and entity consolidation control are aimed at infilling the physical components of a CFS with global subject context (domain area context).

3. Cyber physical computation control and learning management have the following tasks: to add computational monitoring and control, to process big data sets and extract new knowledge about CFS physical components within a domain area in a machine-oriented form.

4. Infographic models management converts a solution (obtained knowledge) from a machine-oriented form to a human-oriented view (infographic modelling). Formation of the CPS under the concept of "Human-Technics-Natural Environment"

5. Analysis and synthesis of infographic models and CPS strategy development.

CPS Infographic models management

When CPS converts the results of its own computations from a machine form into a form convenient for humans, it can be said that the system generates infographic models. This is an important part of CPS management, as it determines the results of the next stage decision-making by the CPS operator through the evaluation of infographic models. Evaluation can be done by analyzing and synthesizing models. An infographic model can be created as a set of infographic slides in forms of dependencies-graphs, charts and other 
visualization methods. The second type of infographic slides are drawings and 3D-views. The third type may be a set of signs / symbols adopted in the society where the CPS managed.

The operator evaluates (using knowledge external to the CFS) the infographic model obtained from the CFS. In one case, he confirms the validity of the model and outputs the component(entity) referenced by the model to the dominant ones. In another case, the operator does not confirm the validity of the model and changes the existing conditions/parameters of the CFS or enters new parameters into the system. In both cases, the CFS receives new knowledge from the operator.

It should be outlined that this evaluation of the infographic model is immanent (inherently relevant) to a particular operator. Different operators can give different estimates under the concept of "Human-Technics-Natural Environment".

The examples of the infographic slides types which can be produced by CPS at the "Infographic models management" level in the domain area of construction are given below.
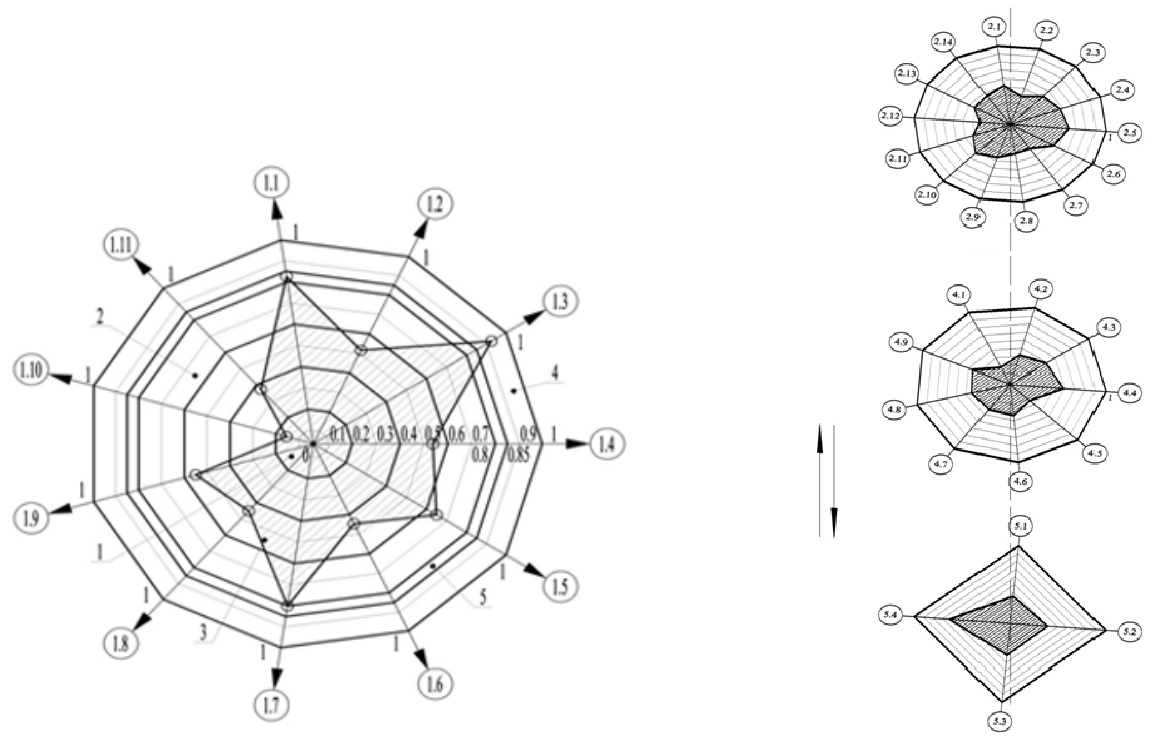

Fig. 3. Flat infographic model (left) and a multilayered model (right) of the CPS parameters of the building under renovation (Losev K.Yu., Chulkov V.O., 2018) [20]

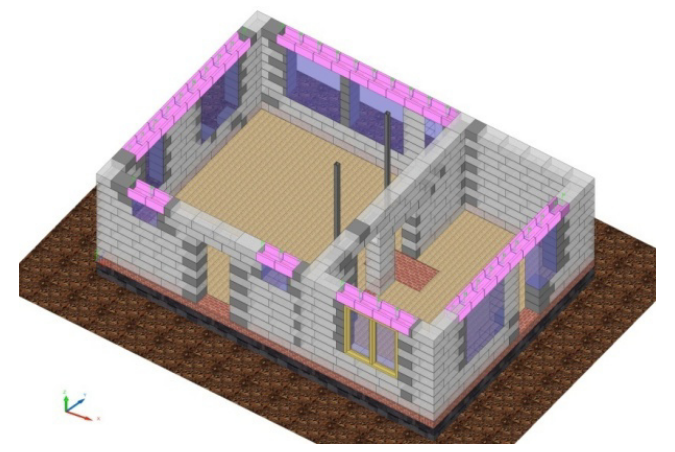

Fig. 4. 3D-views of an object part with infographic message about absence of reinforcement (Losev K., 2017) 


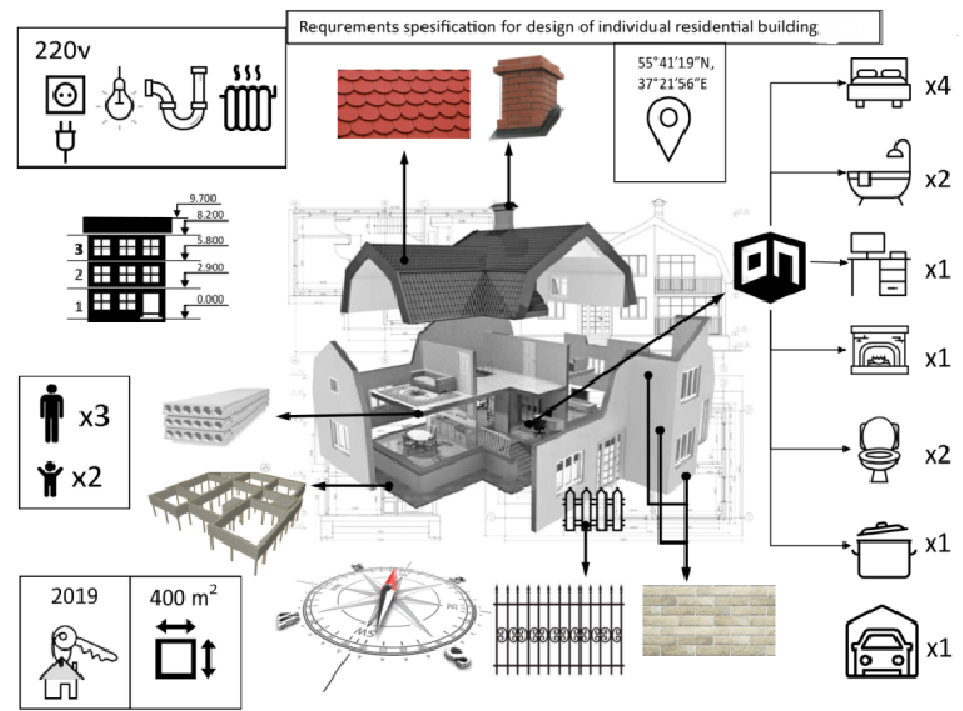

Fig. 5. Infographic structure of signs to form the Employer's Information Requirements (EIR) [21].

\section{Conclusion}

The structure of terms for modern information systems, including approaches, concepts and terms, is proposed as the basis of ontology. The dynamics of the relationship of terms with each other when changing the concept is shown. Model of CPS management structure is presented in accordance with the Industry 4.0 program.

The "Infographic models management" level specific to the CPS is defined as a result of the knowledge conversion from machine-oriented to human-oriented form, which will be the basis for the operator's understanding of the knowledge accumulated by the CPS, and will help to make non-trivial management decisions.

Three types of infographic slides which can be issued by CPS within the domain area of construction and design are proposed.

\section{References}

1. M. Henshaw, OR Insight 19 (3), 51-54 (2016)

2. A. Kaplan, M. Haenlein, Business Horizons, 62 (1), 15-25 (2019)

3. N. Bostrom, Superintelligence: Paths, Dangers, Strategies (Oxford University Press, 2014)

4. Y. Shoham, K. Leyton-Brown, Multiagent Systems: Algorithmic, Game-Theoretic, and Logical Foundations (Cambridge University Press, 2008)

5. G. Weiss, Multiagent systems (Cambridge, The MIT Press, 2013)

6. S. Heath, Embedded systems design. EDN series for design engineers (Newnes, 2003)

7. R.G. Sanfelice, Cyber-Physical Systems: From Theory to Practice, CRC Press, 3-31 (2015)

8. E.A. Lee, S.A. Seshia, Introduction to Embedded Systems, A Cyber-Physical Systems Approach (MIT Press, 2017) 
9. E.A. Lee, The 11th IEEE International Symposium on Object Oriented Real-Time Distributed Computing(ISORC), 363-369 (2008)

10. Th. Fitz, M. Theiler, K. Smarsly, Advanced Engineering Informatics, 41, 100930 (2019)

11. D. Legatiuk, M. Theiler, K. Dragos, K. Smarsly, The 11th International Workshop on Structural Health Monitoring (IWSHM) (Stanford, CA, USA, 2017)

12. K. Marzullo, NSF Cyber Physical Systems (CPS) Program. Past, Present and Future, National Science Foundation (Alexandria, Virginia, USA, 2009)

13. Acetech, Acatech Position Paper, 15-21 (2011)

14. P. Brook, INCOSE International Symposium Edinburgh, Scotland (2016)

15. E. Glaessgen, D. David Stargel, The 20th AIAA/ASME/AHS Adaptive Structures Conference 14th AIAA (2012)

16. J. Reid, D. Rhodes, Conference on Systems Engineering Research, Systems Engineering Advancement Research Initiative (Massachusetts Institute of Technology, 2016)

17. M. Grieves, Digital twin: Manufacturing excellence through virtual factory replication (USA, Michigan LLC, 2014)

18. M. Grieves M., Product Lifecycle Management: Driving the Next Generation of Lean Thinking (New York, McGraw-Hill, 2006)

19. J. Lee, B. Bagheri, H.A. Kao, Manufacturing Letters, 3, 18-23 (2015)

20. K.Yu. Losev, G.O. Chulkov, V.O. Chulkov, IOP Conf. Ser.: Earth Environ. Sci., 177, 012026 (2018)

21. K. Losev, A. Sinenko, IOP Conf. Ser.: Earth Environ. Sci., 90, 012197 (2017) 